\title{
Postoperative Anemia Following Posterior Decompression Surgery for Lumbar Spinal Canal Stenosis
}

\author{
Tatsuro Sasaji, ${ }^{1}$ Kiyoshi Horaguchi, ${ }^{1}$ Nobuhisa Shinozaki, ${ }^{1}$ Noboru Yamada ${ }^{1}$ and \\ Kazuo Iwai ${ }^{1}$
}

${ }^{1}$ Department of Orthopedic Surgery, Fukushima Rosai Hospital, Iwaki, Fukushima, Japan

\begin{abstract}
The incidence of lumbar spinal canal stenosis (LSCS) is increasing in Japan. Posterior lumbar decompression surgery, wide fenestration and laminectomy, for LSCS is a common treatment modality. Compared with posterior fusion surgery, posterior decompression surgery has been considered as less invasive. However, no reports regarding postoperative anemia following posterior decompression surgery have been published. In this retrospective study, we evaluated changes in hemoglobin values following wide fenestration in 80 patients with LSCS based on the number of operated levels, and also analyzed the differences between intraoperative and postoperative bleeding volume. Two patients required allogenic transfusion. The mean preoperative hemoglobin values were $13.6 \mathrm{~g} / \mathrm{dL}$ and the mean postoperative minimum hemoglobin values were $11.2 \mathrm{~g} / \mathrm{dL}$. The mean hemoglobin values decreased by $1.8 \mathrm{~g} / \mathrm{dL}$ in patients with one operated level $(n=15) ; 2.2 \mathrm{~g} / \mathrm{dL}$ in those with two operated levels $(n=31) ; 2.6 \mathrm{~g} / \mathrm{dL}$ in those with three operated levels $(n=23)$; and $3 \mathrm{~g} / \mathrm{dL}$ in those with four operated levels $(n=11)$. The mean decrease in hemoglobin values was calculated as follows: $1.8+0.4 \times(X-1)$, where $X$ was the number of operated levels. The mean intraoperative bleeding volume was $94 \mathrm{ml}$ and the mean postoperative bleeding volume was $418 \mathrm{ml}$. Postoperative bleeding volume was significantly larger than intraoperative bleeding volume. Accordingly, postoperative hemoglobin values can be predicted in patients undergoing wide fenestration. Effective management of postoperative bleeding is necessary to prevent postoperative anemia.
\end{abstract}

Keywords: anemia; hemoglobin; laminectomy; lumbar; spinal stenosis

Tohoku J. Exp. Med., 2013 Jan, 229 (1), 1-4. C 2013 Tohoku University Medical Press

Lumbar spinal canal stenosis is prevalent in elderly people. Japan is an aging society, with increasing incidence of this disorder. Consequently, most patients that require lumbar surgery are elderly; therefore, general control of postoperative condition in this population is important. The main postoperative control points for lumbar surgery are usually infection and bleeding. Fusion surgery is more invasive than decompression surgery. And so, papers regarding postoperative control of fusion surgery have been published. Some detailed reports have been published regarding the inflammatory reactions that occur after spinal instrumentation surgery (Takahashi et al. 2001, 2006). Other reports have addressed the issues of bleeding volume and anemia following posterior fusion surgery (Behrman and Keim 1992; Gause et al. 2008). According to Behrman and Keim (1992), major orthopedic procedures such as spine surgery often entail significant blood loss. Gause et al. (2008) reported that elective posterior spinal surgery necessitates the use of blood for the treatment of postoperative anemia. In both reports, the importance of periopera- tive blood salvage in spine surgery was evident. Some reports have been published regarding the efficacy of intraoperative cell saver blood autotransfusion (Behrman and Keim 1992; Chanda et al. 2002; Gause et al. 2008; Savvidou et al. 2009); however, no reports have examined intraoperative and postoperative bleeding volume along with changes in hemoglobin values following lumbar surgery. To evaluate the efficiency of autotransfusion, we require the basic data for bleeding in lumbar decompression surgery. In this study, differences between the intraoperative bleeding volume and total postoperative bleeding volume, as well as changes in hemoglobin values based on the number of operated levels were analyzed in patients who had underwent posterior lumbar decompression surgery.

\section{Materials and Methods}

We retrospectively reviewed the medical records of 80 consecutive cases ( 37 females and 43 males, aged 55-88 years, mean age 71 years) referred to our institute for surgical treatment between 2010 and 2011 and underwent follow-up examinations for a minimum of

Received September 25, 2012; accepted November 8, 2012. Published online November 27, 2012; doi: 10.1620/tjem.229.1.

Correspondence: Tatsuro Sasaji, Department of Orthopedic Surgery, Fukushima Rosai Hospital, 3-Numajiri, Tsuzura-machi, Uchigo, Iwaki, Fukushima 973-8403, Japan.

e-mail: taturosasaji@ hotmail.com 


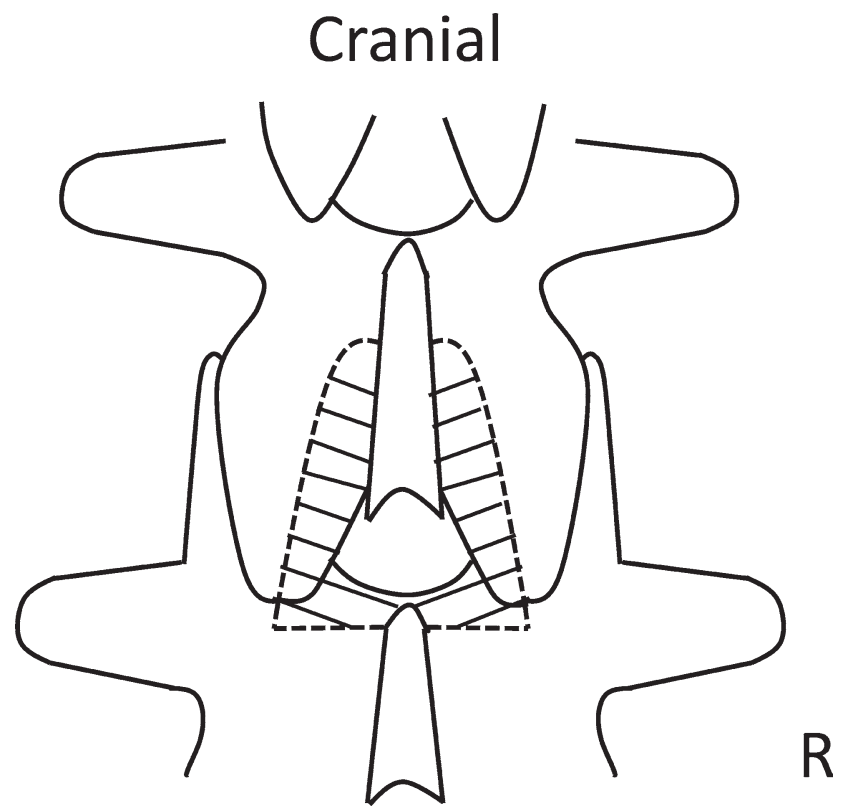

Fig. 1. Surgical schemata displaying wide fenestration (one level).

The shaded area was decompressed. The bilateral medial parts of the inferior facets and the bilateral cranial parts of the lamina and the adjoining ligamentum flavum were removed. The wide fenestration decompressed the dura and nerve roots in the entry zone.

one-year. The mean follow-up period was 16 months (range, 12-28 months). The diagnosis of lumbar spinal canal stenosis (LSCS) was based on clinical symptoms, neurological examinations, and results of magnetic resonance imaging. All the patients had typical neurological symptoms of cauda equina syndrome including bilateral radiating leg pain, numbness, and intermittent claudication. Exclusion criteria for this study included fusion surgery, prior lumbar spinal surgery, foraminal stenosis and intraoperative dural tear. For cases receiving anticoagulant and antiplatelet drug therapy, treatment discontinued and substituted by heparin therapy for several days before surgery. The absolute indication for allogenic transfusion was hemoglobin values of below $8 \mathrm{~g} / \mathrm{dL}$.

All surgeries were performed by a single spinal surgeon. Wide fenestration was performed in all patients (Nakai et al. 1991; Fig. 1); thereafter, continuous suction drains were used after surgery.

Intraoperative bleeding volume along with postoperative bleeding volume on postoperative day 1 , and total postoperative bleeding volume were measured; here, the drainage volume was defined as postoperative bleeding volume. Hemoglobin values were measured the day before surgery and on postoperative days 1, 2, 3, 5, and 7 . Differences between intraoperative bleeding volume and total postoperative bleeding volume were analyzed. The percentage of postoperative acute-phase bleeding was calculated as follows: (bleeding volume on postoperative day 1$) /($ total postoperative bleeding volume $) \times$ 100. A decrease in hemoglobin values was defined as follows: (preoperative hemoglobin values) - (minimum postoperative hemoglobin values). All cases were divided into groups based on the number of operated levels; subsequently, the decrease in hemoglobin values was evaluated.

For statistical purposes, data for bleeding were compared using the Student's $t$-test. A probability value of less than 0.05 was considered significant. Statistical analyses were performed using GraphPad Prism (GraphPad Software Inc., San Diego, CA, USA). All the patients were informed that the data from their cases would be used for this study. The protocol for this study was approved by the Ethics Committee at Fukushima Rosai Hospital (Iwaki, Japan).

\section{Results}

Two of the patients required allogenic transfusion for postoperative anemia. The minimum postoperative hemoglobin values were 7.4 and 7.9 in these cases. The mean intraoperative bleeding volume \pm standard deviation was 94 $\pm 42 \mathrm{ml}$ (range, 14-569 ml), and the mean total postoperative bleeding volume was $418 \pm 189 \mathrm{ml}$ (range, 100-1185 ml; Fig. 2). The mean total postoperative bleeding volume was significantly larger than the mean intraoperative bleeding volume $(P<0.001)$. The mean bleeding volume on postoperative day 1 was $249 \pm 105 \mathrm{ml}$ (range, $40-560 \mathrm{ml}$ ). The mean percentage of postoperative acute-phase bleeding was $61 \pm 12 \%$ (range, 34-94\%). The mean preoperative hemoglobin values were $13.6 \pm 1.6 \mathrm{~g} / \mathrm{dL}$ (range, 8.5-17.6 g/ $\mathrm{dL}$ ) and the mean postoperative minimum hemoglobin values were $11.2 \pm 1.5 \mathrm{~g} / \mathrm{dL}$ (range, 7.4-15.5 g/dL). One level was operated in 15 patients, two in 31 patients, three in 23 patients, and four in 11 patients. Hemoglobin values decreased by $1.8 \pm 0.6 \mathrm{~g} / \mathrm{dL}$ (range, 1-3.2 g/dL) in patients with one operated level, $2.2 \pm 0.6 \mathrm{~g} / \mathrm{dL}$ (range, 1.1-3.7 g/ $\mathrm{dL}$ ) in those with two operated levels, $2.6 \pm 0.8 \mathrm{~g} / \mathrm{dL}$ (range, $1.2-4 \mathrm{~g} / \mathrm{dL}$ ) in those with three operated levels, and $3 \pm 0.8$ $\mathrm{g} / \mathrm{dL}$ (range, 1.6-4.6 g/dL) in those with four operated levels. The mean decrease in hemoglobin values was calculated as follows: $1.8+0.4 \times(\mathrm{X}-1)$, where $\mathrm{X}$ was the number of operated levels (Fig. 3).

\section{Discussion}

Posterior lumbar surgery for LSCS is a common treatment modality in Japan. The types of surgery vary and the levels to be operated differ for each case. For invasive surgeries such as fusion surgery and scoliosis surgery, the management to prevent allogenic transfusion has been examined (Behrman and Keim 1992; Chanda et al. 2002; Brookfield et al. 2008; Gause et al. 2008; Savvidou et al. 2009). However, for less invasive surgery such as decompression surgery, postoperative anemia has not been evaluated in detail. In this study, perioperative anemia, perioperative bleeding volume, and changes in hemoglobin values were evaluated.

The postoperative decrease in hemoglobin values according to the number of operated levels has not been evaluated. In this study, the mean decrease in hemoglobin values according to the number of operated levels was constant. The formula presented above facilitates the prediction of postoperative hemoglobin values in patients who underwent posterior lumbar decompression surgery. The results indicate that management of postoperative anemia is essential if the predicted postoperative hemoglobin values 


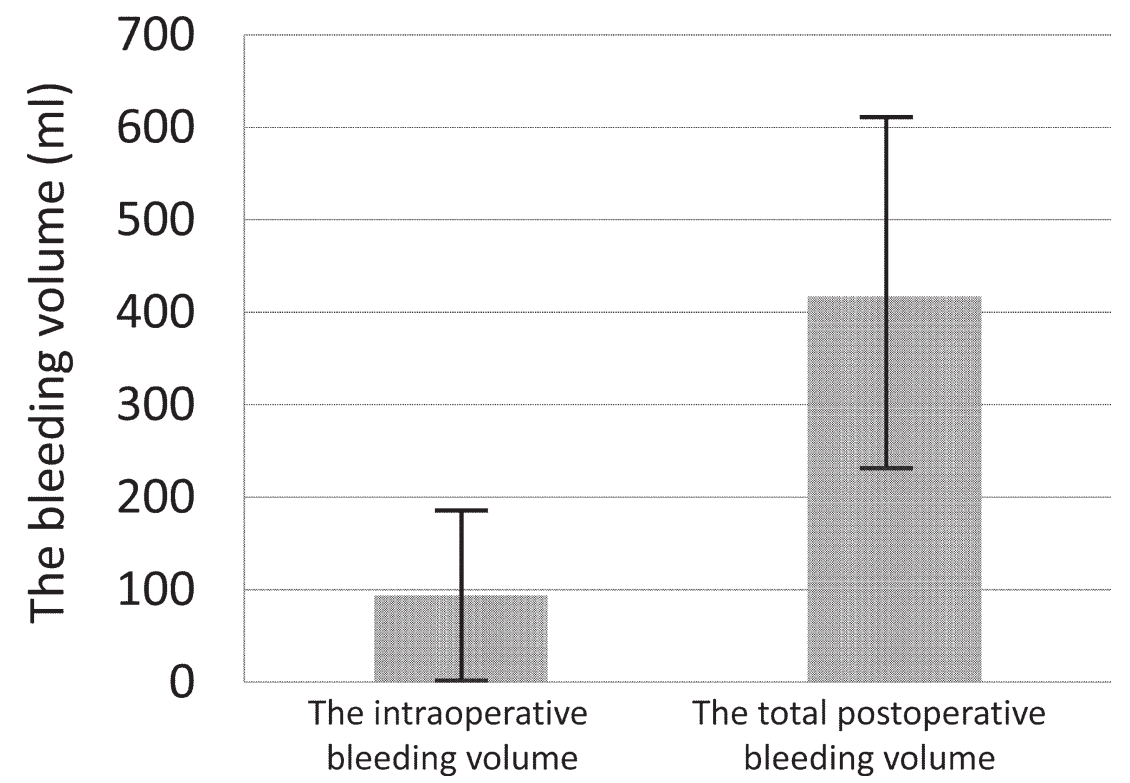

Fig. 2. Comparison of intraoperative and total postoperative bleeding volume.

The mean intraoperative bleeding volume \pm standard deviation was $94 \pm 42 \mathrm{ml}$ (range, 14-569 ml), and the mean total postoperative bleeding volume was $418 \pm 189 \mathrm{ml}$ (range, 100-1,185 ml). The total postoperative bleeding volume was significantly larger than the intraoperative bleeding volume $(P<0.001)$. The error bars represented the standard deviation of the measured bleeding volume.



The number of operated levels

Fig. 3. Changes in hemoglobin values according to the number of operated levels.

The mean decrease in hemoglobin values was $1.8 \pm 0.6 \mathrm{~g} / \mathrm{dL}$ in patients with one operated level, $2.2 \pm 0.6 \mathrm{~g} / \mathrm{dL}$ in those with two operated levels, $2.6 \pm 0.8 \mathrm{~g} / \mathrm{dL}$ in those with three operated levels, and $3 \pm 0.8 \mathrm{~g} / \mathrm{dL}$ in those with four operated levels. The mean decrease in hemoglobin values increased by $0.4 \mathrm{~g} / \mathrm{dL}$ per one level increased. The mean decrease in hemoglobin values was calculated as follows: $1.8+0.4 \times(\mathrm{X}-1)$, where $\mathrm{X}$ was the number of operated levels. The error bars represent the standard deviation of the measured hemoglobin values. 
were below $8 \mathrm{~g} / \mathrm{dL}$.

Previous reports have discussed the efficacy of intraoperative cell saver blood autotransfusion to prevent allogenic transfusion (Behrman and Keim 1992; Chanda et al. 2002; Brookfield et al. 2008; Gause et al. 2008; Savvidou et al. 2009). However, these reports did not compare intraoperative and postoperative bleeding volume. In this study, postoperative bleeding volume was significantly larger than intraoperative bleeding volume. In addition, the bleeding volume on postoperative day 1 accounted for more than half of the total postoperative bleeding volume. To prevent postoperative anemia, management of postoperative hemorrhage is important, especially that occurring on the day of surgery.

No reports on the postoperative anemia following lumbar decompression surgery for LSCS are available. On the basis of present study, we assume that the predicting formula is useful for selecting the appropriate management to prevent postoperative anemia. But, the number of patients was small in the present study. And so, further studies with a large number of LSCS patients would be necessary.

\section{Conclusion}

In cases of posterior lumbar decompression surgery, decrease in hemoglobin values can be predicted before surgery. To prevent postoperative anemia and the need for allogenic transfusion, management of postoperative bleeding is of vital importance.

\section{Conflict of Interest}

The authors declare no conflict of interest.

\section{References}

Behrman, M.J. \& Keim, H.A. (1992) Perioperative red blood cell salvage in spine surgery. A prospective analysis. Clin. Orthop. Relat. Res., 278, 51-57.

Brookfield, K.F., Brown, M.D., Henriques, S.M., Buttacavoli, F.A. \& Seitz, A.P. (2008) Allogeneic transfusion after predonation of blood for elective spine surgery. Clin. Orthop. Relat. Res., 466, 1949-1953.

Chanda, A., Smith, D.R. \& Nanda, A. (2002) Autotransfusion by cell saver technique in surgery of lumbar and thoracic spinal fusion with instrumentation. J. Neurosurg., 96, 298-303.

Gause, P.R., Siska, P.A., Westrick, E.R., Zavatsky, J., Irrgang, J.J. \& Kang, J.D. (2008) Efficacy of intraoperative cell saver in decreasing postoperative blood transfusions in instrumented posterior lumbar fusion patients. Spine, 33, 571-575.

Nakai, O., Ookawa, A. \& Yamaura, I. (1991) Long-term roentgenographic and functional changes in patients who were treated with wide fenestration for central lumbar stenosis. J. Bone Joint Surg. Am., 73, 1184-1191.

Savvidou, C., Chatziioannou, S.N., Pilichou, A. \& Pneumaticos, S.G. (2009) Efficacy and cost-effectiveness of cell saving blood autotransfusion in adult lumbar fusion. Transfus. Med., 19, 202-206.

Takahashi, J., Ebara, S., Kamimura, M., Kinoshita, T., Itoh, H., Yuzawa, Y., Sheena, Y. \& Takaoka, K. (2001) Early-phase enhanced inflammatory reaction after spinal instrumentation surgery. Spine, 26, 1698-1704.

Takahashi, J., Shono, Y., Hirabayashi, H., Kamimura, M., Nakagawa, H., Ebara, S. \& Kato, H. (2006) Usefulness of white blood cell differential for early diagnosis of surgical wound infection following spinal instrumentation surgery. Spine, 31, 1020-1025. 